Sādhanā Vol. 36, Part 3, June 2011, pp. 293-315. (c) Indian Academy of Sciences

\title{
A new hybrid imperialist competitive algorithm on data clustering
}

\author{
TAHER NIKNAM ${ }^{1, *}$, ELAHE TAHERIAN FARD ${ }^{2}$, \\ SHERVIN EHRAMPOOSH ${ }^{3}$ and ALIREZA ROUSTA ${ }^{1,4}$
}

\author{
${ }^{1}$ Marvdasht Branch, Islamic Azad University, Marvdasht, Iran, \\ P.O. Box. 73711-13119 \\ ${ }^{2}$ Shiraz University, Shiraz, Iran, P.O. Box. 71345-1837 \\ ${ }^{3}$ Kerman Graduate University of Technology, Kerman, Iran, P.O. Box. 71315-115 \\ ${ }^{4}$ Department of Electrical and Electronic, Shiraz University of Technology, \\ Shiraz, Iran, P.O. Box. 71555-313 \\ e-mail: taher_nik@yahoo.com; etaherianfard@yahoo.com; \\ s_ehrampoosh@yahoo.com
}

MS received 3 April 2010; revised 31 October 2010; accepted 5 December 2010

\begin{abstract}
Clustering is a process for partitioning datasets. This technique is very useful for optimum solution. $k$-means is one of the simplest and the most famous methods that is based on square error criterion. This algorithm depends on initial states and converges to local optima. Some recent researches show that $k$-means algorithm has been successfully applied to combinatorial optimization problems for clustering. In this paper, we purpose a novel algorithm that is based on combining two algorithms of clustering; $k$-means and Modify Imperialist Competitive Algorithm. It is named hybrid K-MICA. In addition, we use a method called modified expectation maximization (EM) to determine number of clusters. The experimented results show that the new method carries out better results than the ACO, PSO, Simulated Annealing (SA), Genetic Algorithm (GA), Tabu Search (TS), Honey Bee Mating Optimization (HBMO) and $k$-means.
\end{abstract}

Keywords. Modified imperialist competitive algorithm; simulated annealing; $k$-means; data clustering.

\section{Introduction}

Clustering is one of the unsupervised learning branches where a set of patterns, usually vectors in a multi-dimensional space, are grouped into clusters in such a way that patterns in the same cluster are similar in some sense and patterns in different clusters are dissimilar in the same sense. Cluster analysis is a difficult problem due to a variety of ways of measuring the similarity and dissimilarity concepts, which do not have a universal definition. Therefore, cluster seeking

*For correspondence 
is experiment-oriented in the sense that clustering algorithms that can deal with all situations equally well are not yet available. Clustering is not the same as classification. In classification, input samples are labelled but in clustering, they have no initial tag. In fact, with using clustering methods, the same data is specified and implicitly labelled.

Data clustering algorithms can be divided into hierarchical or partitional. In this paper, we focus on the partitional clustering. There is a clustering method called $k$-means. It is the simplest and most commonly used algorithm employing a squared error criterion. In fact, it is a method of cluster analysis which aims to partition $(N)$ observations into $k$ clusters in which each observation belongs to the cluster with the nearest mean $(k<N)$. The number of clusters is estimated by modified EM algorithm. It starts with a random initial partition and keeps reassigning the patterns to clusters based on the similarity between the pattern and the cluster centers until a convergence criterion is met. A major problem with this algorithm is that it is sensitive to the selection of the initial partition and do not guarantee the global optimum value; therefore, it may converge to a local minimum of the criterion function value if the initial partition is not properly chosen.

To overcome this drawback, many clustering algorithms based on evolutionary algorithms such as GA, TS and SA have been introduced. For instance, Kao et al (2008) have proposed a hybrid technique based on combining the $k$-means algorithm, Nelder-Mead simplex search, and PSO for cluster analysis. Cao \& Cios (2008) have presented a hybrid algorithm according to the combination of GA, $k$-means and logarithmic regression expectation maximization. Zalik (2008) has introduced a $k$-means algorithm that performs correct clustering without pre-assigning the exact number of clusters. Krishna \& Murty (1999) have presented an approach called genetic $k$-means algorithm for clustering analysis. Mualik \& Bandyopadhyay (2000) have proposed a genetic algorithm-based method to solve the clustering problem and experiment on synthetic and real life data sets to evaluate the performance. It defines a basic mutation operator specific to clustering called distance-based mutation. Fathian et al (2008) have proposed the HBMO algorithm to solve the clustering problem. A genetic algorithm that exchanges neighbouring centers for $k$-means clustering has presented by Laszlo \& Mukherjee (2007). Shelokar et al (2004) have introduced an evolutionary algorithm based on ACO algorithm for clustering problem. $\mathrm{Ng}$ and Sung have proposed an approach based on TS for cluster analysis (Ng \& Wong 2002; Sung \& Jin 2000). Niknam et al (2008a, 2008b) have presented a hybrid evolutionary optimization algorithm based on the combination of ACO and SA to solve the clustering problem. Niknam et al (2009) have presented a hybrid evolutionary algorithm based on PSO and SA to find optimal cluster centers. Niknam \& Amiri (2010) have proposed a hybrid algorithm based on a fuzzy adaptive PSO, ACO and $k$-means for cluster analysis. Bahmani Firouzi et al (2010) have introduced a hybrid evolutionary algorithm based on combining PSO, SA and $k$-means to find optimal solution.

However, most of evolutionary methods such as GA, TS, etc., are typically very slow to find optimum solution. Recently researchers have presented new evolutionary methods such as ACO, PSO and ICA to solve hard optimization problems, which not only have a better response but also converge very quickly in comparison with ordinary evolutionary methods.

Imperialist competitive algorithm (ICA) is one of the most powerful evolutionary algorithms (Atashpaz-Gargari \& Lucas 2007a, 2007b; Rajabioun et al 2008a, 2008b; Atashpaz-Gargari et al 2008a, 2008b; Roshanaei et al 2008; Jasour et al 2008). It has been used extensively to solve different kinds of optimization problems. This method is based on socio-political process of imperialistic competition. ICA starts with an initial population. In this algorithm any individual of the population is called a country. Some of the best countries in the population are selected to be the imperialist states and all the other countries form the colonies of these imperialists. After dividing all colonies among imperialists and creating the initial empires, these colonies start 
moving toward their relevant imperialist country. This movement is a simple model of assimilation policy that was perused by some imperialist states. The movement of colonies toward their relevant imperialists along with competition among empires and also collapse mechanism will hopefully cause all the countries to converge to a state in which there exist just one empire in the world and all the other countries are its colonies. As a result, ICA could be taken into account as a powerful technique. Nevertheless, it may be trapped in local optima especially when numbers of imperialists increase. To alleviate this drawback, mutation can help to divert the movement of colonies toward their relevant imperialist into new positions. Also we use the simulated annealing (SA) as a local search around the best solution found by MICA algorithm. This approach provides better opportunity of exploring for colonies. To use the benefits of $k$-means and ICA, and reduce their disadvantages a novel hybrid evolutionary optimization method, called hybrid K-MICA is presented in this paper, for optimum clustering $(N)$ objects into $k$ clusters. This hybrid algorithm not only has a better response but also converges more quickly than ordinary evolutionary algorithms. In this method, after generating initial countries, $k$-means is applied to improve the position of colonies.

The paper is organized as follows: In section 2, the cluster analysis problem is discussed. In sections 3 and 4, imperialist competitive algorithm and simulated annealing are described, respectively. In addition, in sections 5-7, modified MICA, the hybrid K-MICA and application of hybrid K-MICA in clustering are shown, respectively. In section 8, the feasibility of the hybrid K-MICA is demonstrated and compared with K-MICA, MICA-K, MICA, ICA, ACO, PSO, SA, GA, TS, HBMO and $k$-means for different data sets. Finally, section 9 includes a summary and the conclusion.

\section{2. $k$-Means algorithm}

The term ' $k$-means' was first used by MacQueen (1967), though the idea goes back to Hugo Steinhaus in 1956. Lloyd (1982) first proposed the standard algorithm in 1957 as a technique for pulse-code modulation, though it was not published until 1982. It is one of the most popular methods of clustering.

The goal that the algorithm prepends is to minimize the total cost function, which is a squared error function. Each cluster is identified by a centroid. The algorithm follows an iterative procedure. Initially, $k$ cluster is created randomly. Next, the centroid of each group (cluster) is computed. After this, a new partition is built by associating each entry point to the cluster whose centroid is closest to it. Finally, the cluster centers are recalculated for the new clusters. The algorithm is executed until convergence is reached. The cost function is calculated as follows:

For each data vector, assign the vector to the cluster with the closest centroid vector, where the distance to the centroid is determined using

$$
F(X, Y)=\sum_{i=1}^{N} \min \sum_{j=1}^{n}\left(x_{i, j}-y_{i, j}\right)^{2},
$$

where $X$ denotes the input data vector, $Y$ denotes the centroid vector of cluster, $n$ subscripts the number of features of each centroid vector and $(N)$ the number of data input. Figure 1 shows its flowchart.

There are two problems in $k$-means as given below:

(i) It is unclear how to choose the initial centers of clusters.

(ii) Number of clusters need to be known in advance. 


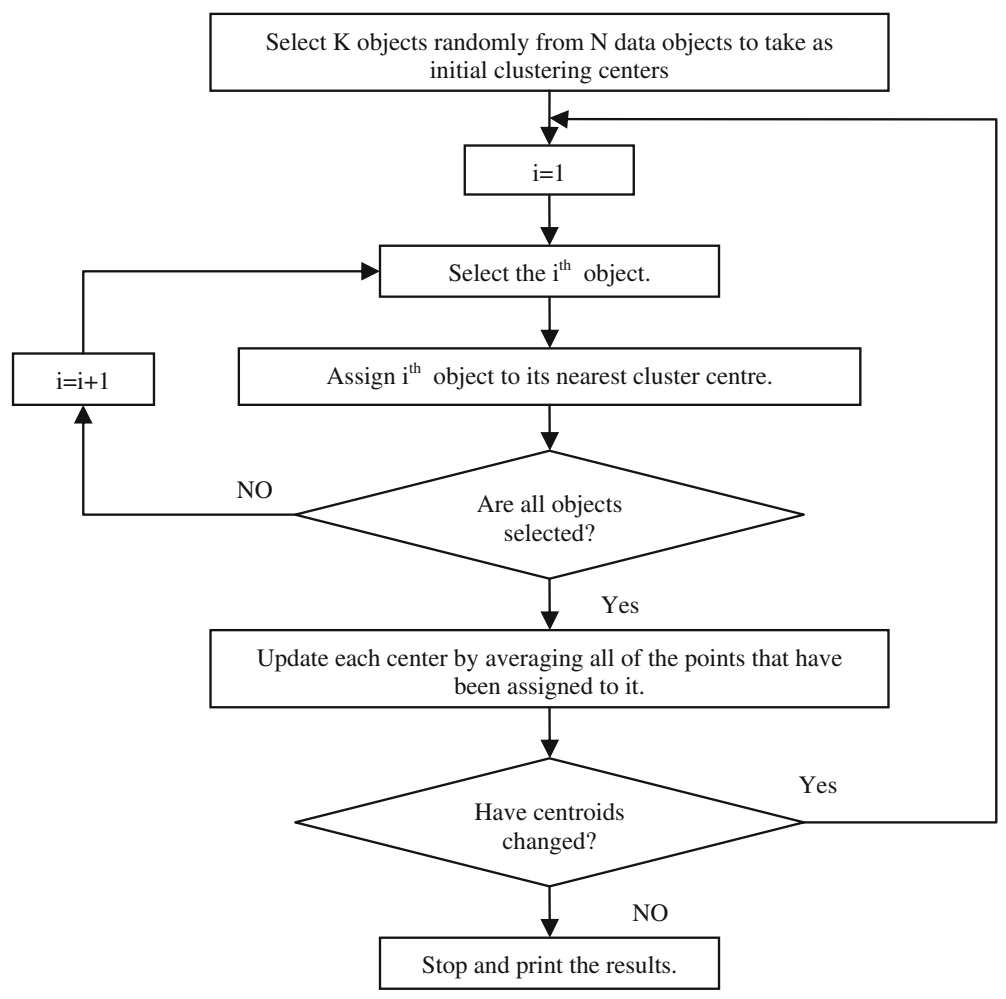

Figure 1. Flowchart of $k$-mean.

As $k$-means is an investigative method, there is no guarantee that it will converge to the global optimum, and the result may depend on the initial clusters. Because of the fact that the algorithm is usually very fast, it is common to run it several times with different starting conditions. However, reaching the convergence can be very slow. For solving this problem, ICA is employed to choose initial centers of clusters. The number of cluster centers $k$, can be known in advance. In practical data sets, it is impossible to determine what the best number of clusters is. Therefore, there are some different methods to find $k$ (Safarinejadian et al 2010; Figueiredo \& Jain 2002; Tibshirani et al 2001). We select one of the approaches proposed by Figueiredo \& Jain (2002).

The EM (Expectation Maximization) algorithm is used in mathematical statistics in order to find estimates of the maximum likelihood parameters of probabilistic models, when the model depends on some hidden variables. Each iteration of the algorithm consists of two steps. In the E-step (expectation) the expected value of the likelihood function is calculated, and the latent variables are treated as observed. In the M-step (maximization), the maximum likelihood is estimated, thus increasing the expected likelihood that is calculated in the E-step. Then, this value is used for the E-step to the next iteration. This algorithm will continue until it converges to the answer.

To achieve this goal, the modified EM algorithm has been used (Figueiredo \& Jain 2002). First, we initialize the modified algorithm with $(N)$ clusters, which is above the numbers we knew to be presented. Then this algorithm is executed until convergence is reached. At this moment, the weight of each cluster represents the number of data entities associated to that cluster. Next, the 


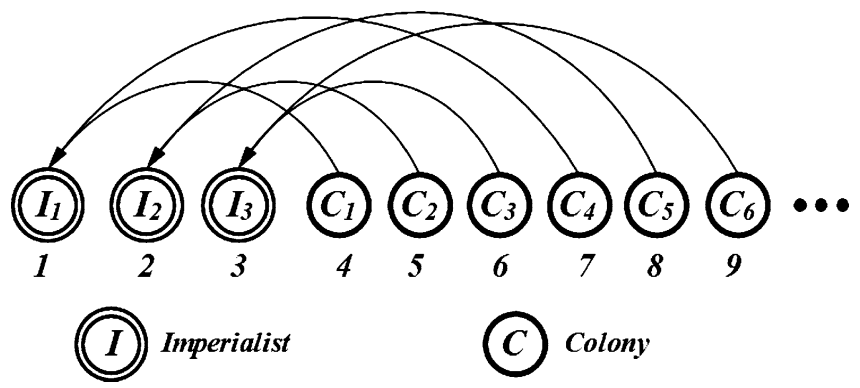

Figure 2. Divide colonies among imperialist.

cluster with zero weight will be removed. This producer will be gone on until finding the proper number of clusters. In this paper, the number of clusters is estimated by modified EM algorithm.

\section{Original ICA}

Imperialist competitive algorithm (ICA) is one of the most powerful evolutionary algorithms. It has been used extensively to solve different kinds of optimization problems. This method is based on socio-political process of imperialistic competition. ICA is started with an initial population. In this algorithm, each member of the population is called a country. Some of the best countries in the population are selected to be the imperialist states and all the other countries form the colonies of these imperialists. All the colonies of initial population are divided among the mentioned imperialists based on their cost function, respectively; for instance, we assume the number of imperialists is three. In this case, the forth to sixth countries will be considered as the first colony of the empires. After that, the seventh to the ninth countries will be selected respectively as the second colony of the empires. This action will be continued to the entire countries. This conception is illustrated in figure 2 .

After all empires were formed, the competition between countries begins. First, the colonies in each of empires start moving toward their relevant imperialist state and change the place in the new position. This movement is shown in figure 3. In this model, $a$ is a random variable with

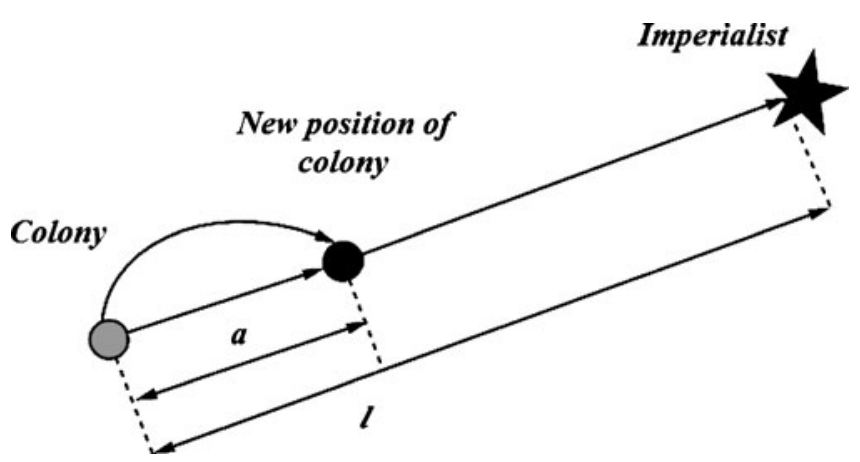

Figure 3. Moving colonies toward their related imperialist. 


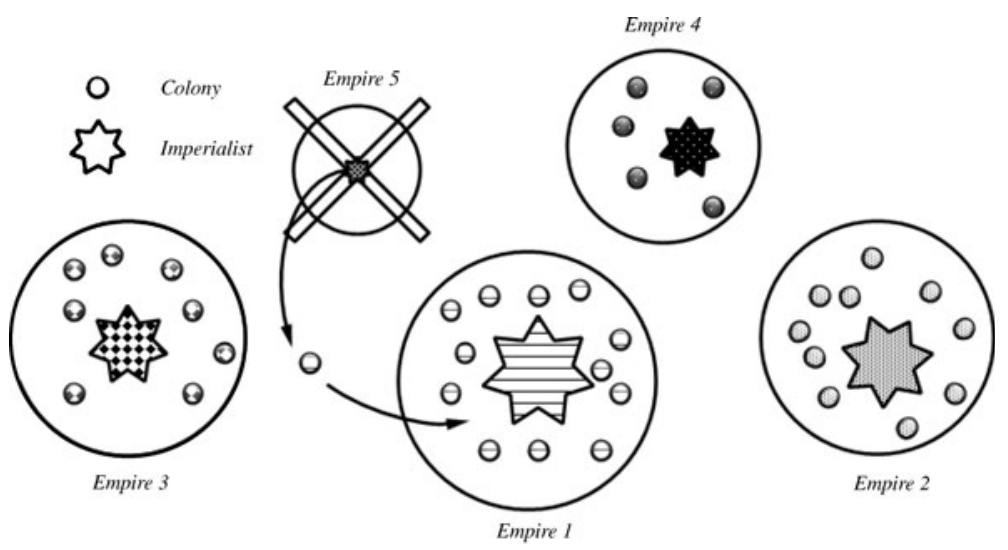

Figure 4. Elimination of the weakest empire.

uniform distribution and $\beta$ is a number that is greater than one (in most of implementations, $\beta$ is equal to 2). Also, $l$ is supposed the distance between the colony and the imperialist.

$$
a \sim U(0, \beta \times l)
$$

During this movement, if the colony gets higher cost than its imperialist does, they will exchange their positions. After that, the algorithm will be continued with the new imperialist. The power of each empire is made up of imperialist cost function and colonies. Competition among empires is the most important part of ICA. It is based on empires power. In this case, the empire which is weaker than the others, loses its colonies until there will be no colony in that. The result of this action is extinction of the weakest empire. After that, its imperialist is considered as the best empire colony. This conception is depicted in figure 4.

The final level of imperialist rivalry is when there is only one empire in the world. This is the optimum point. ICA flowchart is expressed in figure 5.

\section{Simulated annealing}

Simulated annealing is a generalization of a Monte Carlo method for examining the equations of state and frozen states of n-body systems. The concept is based on the manner in which liquids freeze or metals recrystalise in the process of annealing. In an annealing process, a melt is initially at high temperature, disordered, and is slowly cooled so that the system at any time is approximately in thermodynamic equilibrium. As cooling proceeds, the system becomes more ordered then approaches a 'frozen' ground state at zero temperature. Hence, the process can be thought of as an adiabatic approach to the lowest energy state. If the initial temperature of the system is too low, the system may become quenched (Niknam et al 2008a).

The original Metropolis scheme was that an initial state of a thermodynamic system was chosen at energy E and temperature T. Holding T constant, the initial configuration is perturbed and the change in energy, $\Delta E$, is computed. If the change in energy is negative, the new configuration is accepted. If the change in energy is positive, it is accepted with a probability factor $\exp (-\Delta E / T)$. This process is then repeated sufficient times to give good sampling statistics for 


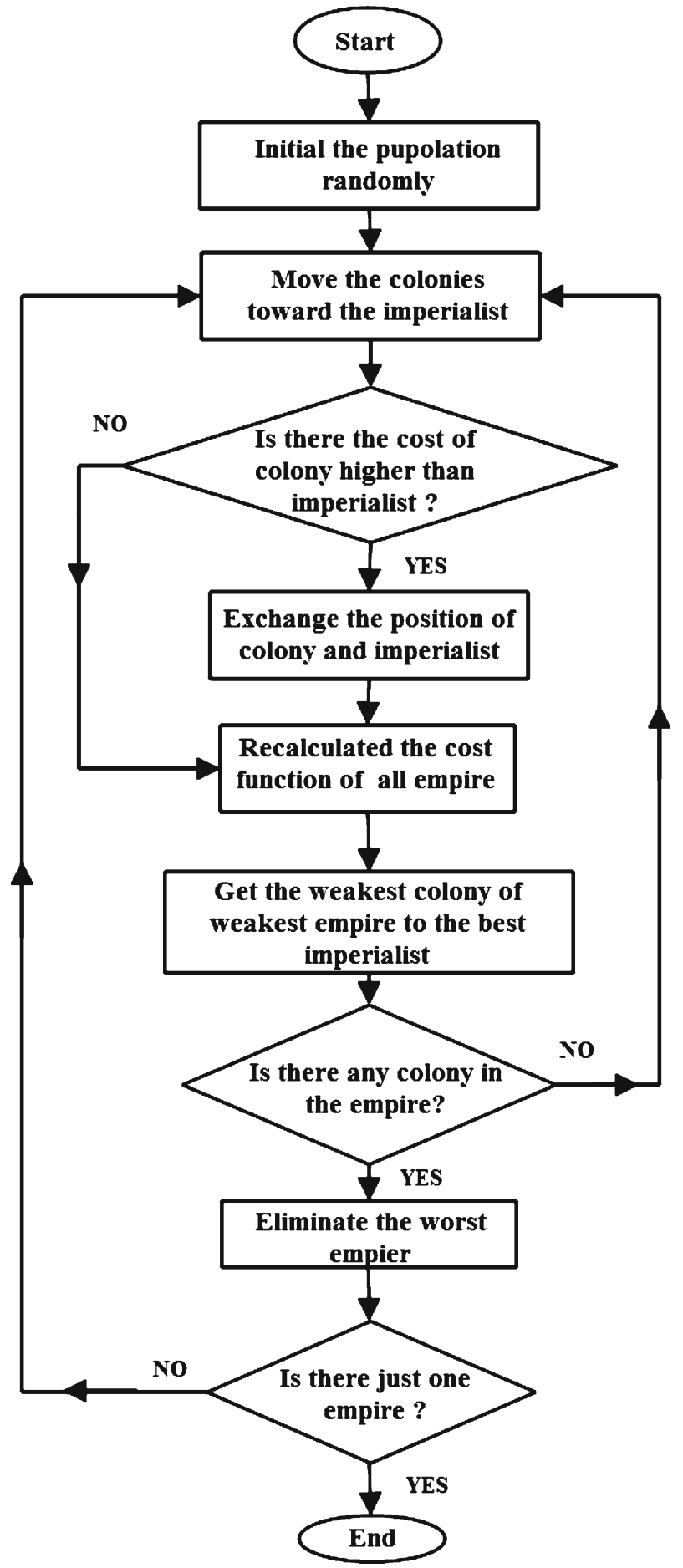

Figure 5. Imperialist competitive algorithm (ICA) flowchart. 
the current temperature, and then the temperature is decremented and the entire process repeated until a frozen state is achieved at $\mathrm{T}=0$.

The general procedure for the SA algorithm can be summarized as follows (Bahmani Firouzi et al 2010):

Step 1: Select an initial solution $\mathrm{X}$ and an initial temperature $\mathrm{T}$.

Step 2: Find another solution, namely $X_{\text {next }}$, by modifying the last answer X.

Step 3: Calculate the energy differential $\Delta E=f\left(X_{\text {next }}\right)-f(X)$.

Step 4: If $\Delta E<0$ go to Step 9.

Step 5: Generate a random number, namely $\mathrm{R}$, between 0 and 1 .

Step 6: If $R<\exp \left(-\frac{\Delta E}{T}\right)$ go to Step 9 .

Step 7: Repeat Steps 2-6 for a number of optimization steps for the given temperature.

Step 8: If no new solution, $X_{\text {next }}$ is accepted then go to Step 10.

Step 9: Decrease the temperature T, replace $X$ with $X_{\text {next }}$ and go to Step 2.

Step 10: Reheat the environment with setting $T$ to a higher value.

Step 11: Repeat Steps 1 through 10 until no further improvement obtained.

\section{Modified ICA}

In order to improve the convergence velocity and accuracy of the ICA algorithm, this paper proposes a new mutation operator. Premature convergence may occur under different situations:

(i) The population has converged to local optimum of the objective function.

(ii) The population has lost its diversity.

(iii) The search algorithm has proceeded slowly or has not proceeded at all.

Mutation is a powerful strategy to diversify the ICA population and improve the ICA's performance in preventing premature convergence to local minima. The used mutation is described as follows:

Let $X$ illustrate the position for each country in an N-dimensional problem. At first, 10 individuals are generated randomly according to the following equation:

$$
\begin{aligned}
& X_{\text {mut }, i}=X+\varepsilon \quad i=1,2,3, \ldots, 10 \\
& \varepsilon=\left[\begin{array}{llll}
\varepsilon_{1} & \varepsilon_{2} & \ldots & \varepsilon_{n}
\end{array}\right]_{1 \times n} \\
& \varepsilon_{i}=\operatorname{rand}(.) .
\end{aligned}
$$

If the best solution among $X_{m u t, i}$ is better than $X$, it replaces $X$. Also in the proposed algorithm, the SA algorithm has updated the best solution found by MICA, which is the best local search algorithm. In other words, in the hybrid algorithm, at each iteration, the best solution value is considered as an initial point of the SA algorithm.

\section{Hybrid K-MICA}

As it was said before, $k$-means is one of clustering algorithms, which can be implemented easily. However, it has some disadvantages. One of them is that its results depend on initial values. Another one is that it converges to local minimum. Recently, numerous ideas have been used 
to alleviate this drawback by using global optimization algorithms such as GA (Krishna \& Murty 1999), TS (Ng \& Wong 2002), PSO and hybrid K-PSO (Kao et al 2008), hybrid PSOSA (Niknam et al 2008a, 2008b), hybrid PSO-ACO-K (Bahmani Firouzi et al 2010), HBMO (Fathian et al 2007), hybrid ACO-SA (Niknam et al 2008a, 2008b) and PSO-SA-K (Morales \& Erazo 2009). The aim of this paper is using the combination method to solve these problems. As a result, we merge both $k$-means and MICA methods.

There are different methods for combination $k$-means with MICA. In the first case, $k$-means is used to generate the population and its output initializes MICA. The second type, MICA

Begin

Generate an initial population randomly

Estimate the number of $k$ cluster

Calculate the objective function for the initial population

Sort the initial population based on their objective function values and select the imperialist states

Divide colonies among imperialist

Use $k$-means algorithm for each empire

Do \{

Place one colony as initial $k$ centroids object that are clustered.

Do \{

Assign each object to the group that has the closest centroid

Recalculate the positions of the $k$ centroids

\}

While (the centroids no longer move)

\}

While (all colonies selected)

Do \{

Do \{

Select the $i^{\text {th }}$ empire

Do \{

Select the $j^{\text {th }}$ colony

Move the colony toward its imperialist state

Use mutation to change the direction of colony

Calculate the objective function value for the two new populations

Compare both new cost and select the best one

Replace ${ }^{\text {th }}$ colony with new one

\}

While (all colonies selected)

Sort all colonies of $j^{\text {th }}$ empire based on their cost functions

Check cost of all colonies in each empire

if there is a colony which has a lower cost than its imperialist

End if

Exchange the position of the colony and the imperialist

Update the position of the $i^{\text {th }}$ empire

\}

While (all empires selected)

Calculate total cost of empires

Find the weakest empire

Give one of its colonies to the winner empire

Check the number of colony in each empire

If there is an empire without colony

Remove empire and give its imperialist to the best empire

End if

Run the SA algorithm while the best solution is considered as its initial point If the output of SA is better than the best solution Swap the best solution and the SA output

\}

While (there is more than one empire)

End

Figure 6. Pseudo code of hybrid K-MICA. 
initializes the population and competition will be done; the last remaining empire will be given to $k$-means. Moreover, in the last one, population is generated with MICA, initial empires form, and then $k$-means is applied to improve the position of empire's colonies and imperialists. The

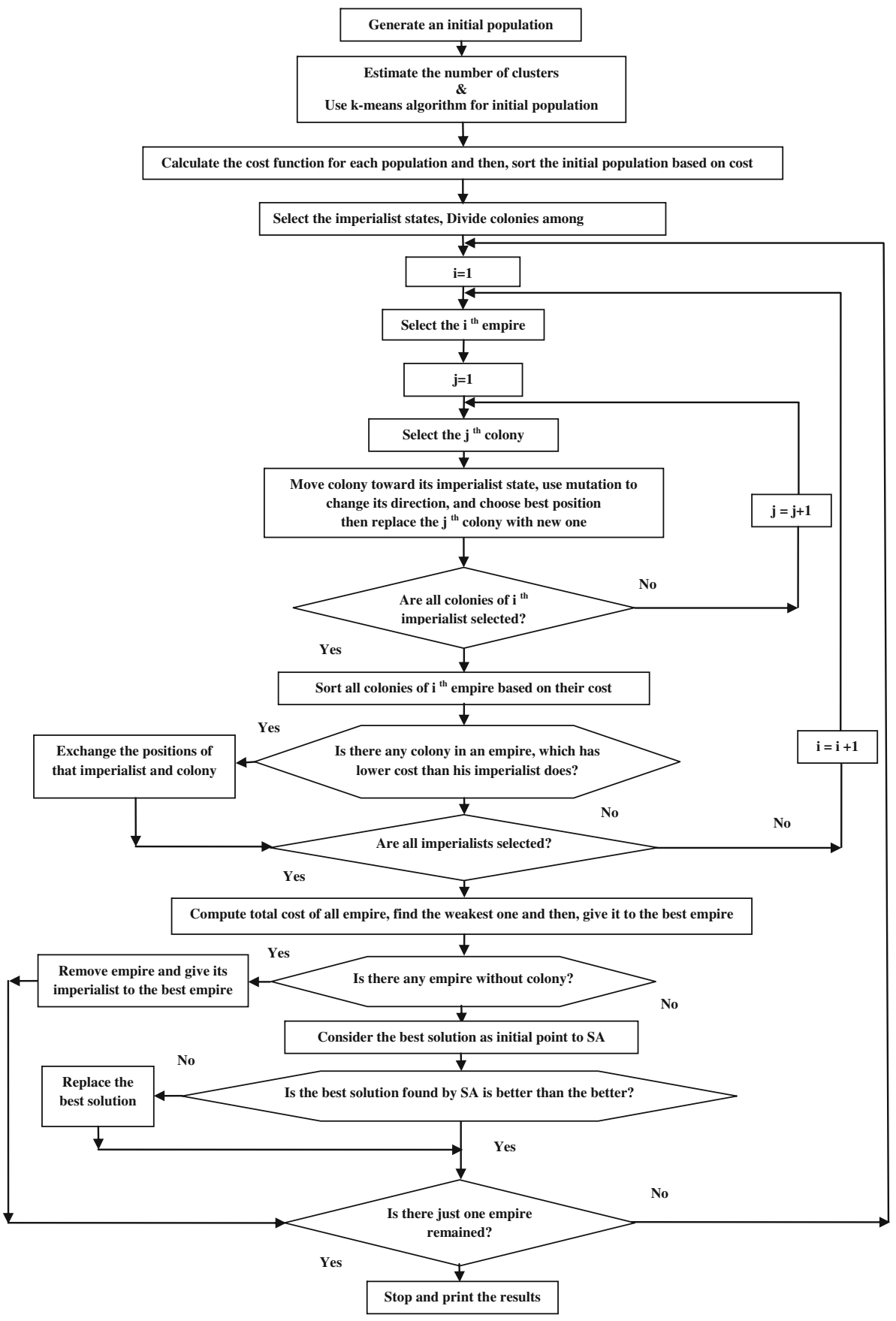

Figure 7. K-MICA flowchart. 
result of this algorithm will be given to MICA, again. Although the results of these procedures are better than the MICA, however, the convergence speed and accuracy of the last one is the best.

The final algorithm is called hybrid K-MICA. According to original ICA, first, the primary population generates and then empires with their possessions take place. Applying $k$-means to each empire causes us to improve the initial population of colonies. It makes the hybrid algorithm converge more quickly and prevents it from falling into local optima. The outputs of $k$-means form the initial empires of modified ICA. To improve the income of algorithm, it is better not to remove the powerless imperialist when it loses all possessions. This imperialist is one of the best answers and can be contributed in imperialistic competition as a weak colony or to be given to the powerful empire. Pseudo-code of hybrid K-MICA and flowchart of K-MICA are shown in figures 6 and 7, respectively.

\section{Application of hybrid K-MICA on clustering}

There are some input data, which would be clusters and the control variables that are referred to the cluster centers. In this part, the hybrid K-MICA application on clustering problem is shown in some steps as follows:

Step 1: Generate an initial population and then Estimate the number of $k$ cluster

An initial population of input data is created randomly:

$$
\begin{aligned}
& \text { Population }=\left[\begin{array}{c}
X_{1} \\
X_{2} \\
\ldots \\
X_{N_{p o p}}
\end{array}\right] \\
& X_{i}=\text { Country }_{i}=\left[\text { Center }_{1}, \text { Center }_{2}, \ldots, \text { Center }_{k}\right] i=1,2, \ldots, N_{p o p} \\
& \text { Center }_{j}=\left[x_{1}, x_{2}, \ldots, x_{d}\right] j=1,2, \ldots, k \\
& x_{j}^{\min }<x_{j}<x_{j}^{\max },
\end{aligned}
$$

where $X_{i}$ is one of the countries, Center ${ }_{j}$ is $j^{\text {th }}$ cluster center for $i^{\text {th }}$ country, $d$ is the dimension of each cluster center, $N_{\text {pop }}$ is the number of population, and $(k)$ is the number of clusters. $x_{j}^{\max }$ and $x_{j}^{\min }$ (each feature of center) are the maximum and minimum values of each point referring to the $j^{\text {th }}$ cluster center. By using modified EM algorithm, $k$ will be estimated.

\section{Step 2: Calculate objective function value}

The cost function is evaluated for each country as below:

$$
\begin{gathered}
F(X)=\sum_{m=1}^{N}\left(\sqrt{\left(\text { Center }_{1}-Y_{m}\right)^{2}+\left(\text { Center }_{2}-Y_{m}\right)^{2}+\ldots+\left(\text { Center }_{K}-Y_{m}\right)^{2}}\right) . \\
(N=\text { number of input data })
\end{gathered}
$$


Step 3: Sort the initial population based on the cost function values

Initial population should be sorted ascending based on cost function. Select $N_{c o l}$ of the sorted population as follows:

$$
\begin{aligned}
& X_{-} \text {sort }=\left[\begin{array}{cc}
X_{s, 1} & F_{s, 1} \\
X_{s, 2} & F_{s, 2} \\
\cdot & \cdot \\
\cdot & \cdot \\
X_{s, N_{c o l}} & F_{S, N_{c o l}}
\end{array}\right]_{\left(N_{c o l}\right) \times(n+1)} \\
& N_{\text {col }}=\left(N_{\text {pop }}-N_{\text {imp }}\right) \\
& (n=\text { number of parameters }),
\end{aligned}
$$

where, $X_{s, 1}$ and $X_{s, N_{c o l}}$ are the vertices with the lowest and the highest function values, respectively. $F_{S, 1}$ and $F_{S, N_{c o l}}$ represent the corresponding observed function values, respectively. $N_{c o l}$ is the number of colonies. $N_{i m p}$ is the number of imperialists. $N_{\text {col_imp }}$ is the number of colonies in each imperialist.

\section{Step 4: Select the imperialist states}

Countries with minimum cost function are chosen as the imperialist states and the remaining ones form the colonies of these imperialists.

\section{Step 5: Divide colonies among imperialist}

After selecting the imperialists, the remaining countries are assigned to the imperialists based on their cost function value, respectively.

In this method, after sorting the countries due to their cost function, each colony is divided into imperialists. These imperialists search the space independently and in different directions to evolve the solutions. The colonies are portioned as follows:

Matrix $X \_s o r t$ is partitioned into $N_{i m p}$ imperialists $Y_{1}, Y_{2}, \ldots . Y_{N_{i m p}}$, each containing $N_{c o l}$ colonies, such that

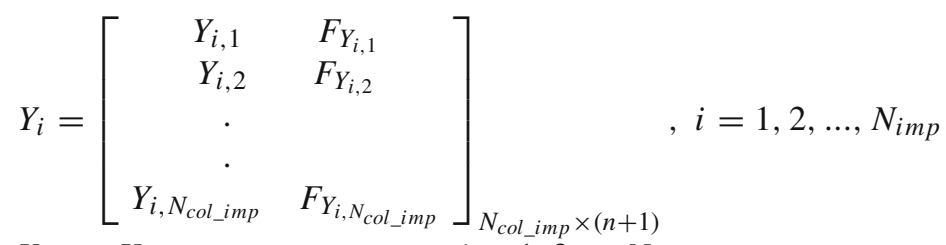

$$
\begin{aligned}
& Y_{i, j}=X_{s,\left(i+N_{i m p} \times(j-1)\right)}, \quad j=1,2, . ., N_{\text {col_imp }} \\
& F_{Y_{i, j}}=F_{Y_{i},\left(i+N_{i m p} \times(j-1)\right)},
\end{aligned}
$$

For example, for $N_{i m p}=3, X_{s, 1}$ goes to imperialist $1\left(Y_{1}\right), X_{s, 2}$ goes to imperialist $2\left(Y_{2}\right), X_{s, 3}$ goes to imperialist $3\left(Y_{3}\right), X_{s, 4}$ goes to imperialist $1\left(Y_{1}\right)$, and so on.

\section{Step 6: Use k-means algorithm for each empire}

- Select an empire and choose one of its colonies, which represent initial group centroids.

- Assign each object (which has to be clustered) to the group that has the closest centroid.

- Recalculate the positions of the centroids.

- Go to step 3 and 4 until the centroids no longer move and these new centroids replace the old colony.

- Repeat step 1 to 6 for all empires. 
Step 7: Move colonies toward their imperialist states

Step 8: Use mutation to change the direction of colonies as it is described in modified ICA clearly Step 9: Check the cost of all colonies in each empire

As it is mentioned, during the colonies movement toward the imperialist, some of them may get better position to reach the imperialist. Indeed, it will be happened when colonies cost function is less than imperialists.

\section{Step 10: Check total cost of each empire}

It is possible to calculate each empire cost which is related to the power of both imperialist and its colonies. It is calculated as follows:

$$
\begin{aligned}
& \text { T. } C_{n}=F\left(\text { imperialist }_{n}\right)+\xi \text { mean }\left\{F\left(\text { colonies of empire } e_{n}\right)\right\} \\
& (0<\xi<1) .
\end{aligned}
$$

$T . C_{n}$ is the total cost of $n^{\text {th }}$ empire. In addition, $\xi$, as an attenuation coefficient, is used to reduce the effect of colonies cost $(0<\xi<1)$.

\section{Step 11: Do imperialistic competition}

The probabilities assigned to the countries in the empire are inversely proportional to their cost. A country with the lowest cost has the greatest probability of being imperialist, while the country with the highest cost has the lowest probability of being imperialist. A random number determines which country is selected. This type of weighting is often referred to as Roulette wheel weighting. There are two techniques: rank weighting and cost weighting. We focused on Roulette wheel cost selection.

In cost weighting method, the probability of selection is calculated from the cost of the country rather than its rank in the population. Initially, a normalized cost is calculated for each country by subtracting the lowest cost of the discarded countries $\left(T . C_{N_{i m p}+1}\right)$ from the cost of all the countries in the empire:

$$
T . C_{n}^{n o r m}=T . C_{n}-\min \cos t_{N_{i m p}+1}\left\{T . C_{N_{i m p}+1}\right\} .
$$

Subtracting $\left(T . C_{N_{i m p}+1}\right)$ ensures all the costs are negative.

$$
p_{p_{n}}=\left|\frac{T \cdot C_{n}^{n o r m}}{\sum_{n=1}^{N_{i m p}} T \cdot C_{n}^{\text {norm }}}\right|
$$

where $T . C_{n}^{\text {norm }}$ is normalized total cost of the $n^{\text {th }}$ empire and the possession probability of each empire is $p_{p_{n}}$. Next, it is necessary to calculate cumulative probability as:

$$
\begin{aligned}
C_{P_{l}} & =P_{P_{l}} \\
C_{p_{n}} & =\sum_{i=1}^{n} p_{p_{l} .} .
\end{aligned}
$$

According to this equation cumulative probability for the last $n$ is equal to one.

After that, a random number with uniform distribution generates and compares with all $C_{P n}$. Each sector with higher probability will have more chance to be chosen. Therefore, the winner 
empire will be specified. This approach tends to weight the top country more when there is a large spread in the cost between the top and bottom country. The probabilities must be recalculated in each generation. After realizing the winner empire, the weakest colony of the weakest empire will be given to the winner one. Then we should subtract one of the populations of this weak empire and add one to the winner's population.

\section{Step 12: Remove weakest empire}

Powerless empires will collapse in the imperialistic competition and their colonies will be divided among other empires. In modelling collapse mechanism, different criteria can be defined for considering an empire powerless. In most of our implementation, when we assume an empire collapsed, we eliminate it as it loses all of its colonies.

\section{Step 13: Run the SA algorithm}

Simulated annealing will be run while the best solution is considered as its initial point. If the output of SA is better than the best solution, swap the best solution and the SA output.

\section{Step 14: Check number of empire}

If there is just one empire, stop it, otherwise return to step 7.

\section{Experimental results}

In this section, the scope is to compare the hybrid K-MICA clustering algorithm with several typical stochastic algorithms including K-MICA, MICA-K, MICA, ICA, ACO, PSO, SA, GA, TS, HBMO and $K$-means. We use four real-life data sets (Iris, Wine, Vowel and Contraceptive Method Choice $(C M C)$ ), and a manufactured data set. They are described as follows:

\subsection{Iris data $(N=150, d=4, K=3)$}

This data set includes 150 random samples of flowers from the iris species setosa, versicolor, and virginica collected by Anderson (1935). For each species there are 50 observations including sepal length, sepal width, petal length, and petal width in centimeters. This data set was used by Fisher (1936) in his initiation of the linear-discriminant-function technique (Bahmani Firouzi et al 2010).

\subsection{Wine data $(N=178, d=13, K=3)$}

This is the wine data set, which is also taken from MCI laboratory. These data are the results of a chemical analysis of wines grown in the same region in Italy extracted from three different cultivars. There are 178 instances with 13 numeric attributes in wine data set. All attributes are continuous. There are no missing attribute values (Bahmani Firouzi et al 2010).

\subsection{Contraceptive method choice $(N=1473, d=10, K=3)$}

This data set is a subset of the 1987 National Indonesia Contraceptive Prevalence Survey. The samples were married women who either were not pregnant or did not know if they were at 
the time of interview. The problem is to predict the current contraceptive method choice (no use of long-term methods, or short-term methods) of a woman based on her demographic and socio-economic characteristics (Bahmani Firouzi et al 2010).

\subsection{Vowel data $\operatorname{set}(N=871, d=3, K=6)$}

This data set consists of 871 patterns. There are six overlapping vowel classes and three input features (Bahmani Firouzi et al 2010).

\subsection{Manufactured data set}

This data set consists of 10000 patterns. Milling is one of the most versatile machining processes and it can be product of different shapes including flat surfaces, slots, and contours. Milling machine classification is based on design, operation, and/or purpose. In this part, five models of milling machines can be studied as follow (Huan Min Xu \& Dong Bo Li 2008):

- Vertical Milling Machine (VMM)

- Horizontal Milling Machine (HMM)

- Single \& Dual-Face modular Milling Machine (SDFMM)

- Plano-Milling Machine (PMM)

- Single \& Double Column surface Milling Machine (SDCMM).

For these models, we select six attributes, each attribute in the set is denoted by its corresponding element:

- WT: Maximum weight of work piece (unit: kilogram or $\mathrm{kg}$ )

- PW: Power (unit: kilowatt or kw)

- SR: Surface roughness (unit: $\mu \mathrm{m}$ )

- FS: Flatness (unit: $\mathrm{mm}$ )

- SS: Maximum speed of spindle (unit: rpm)

- FT: Maximum feeds of table (unit: $\mathrm{mm} / \mathrm{min}$ ).

$N_{\text {pop }}, N_{\text {imp }}, \beta, \xi, \gamma$ are the parameters which are needed for implementation of the hybrid K-MICA that are shown in table 1.

The results of 10 runs of the algorithm for iris data set with different parameters are depicted in the table 2. It illustrates this method is invariant about variable parameters.

The algorithms are implemented by using MATLAB 7.6 on a Pentium IV, $2.8 \mathrm{GHz}, 2 \mathrm{~GB}$ RAM computer.

A comparison among the results of hybrid K-MICA, K-MICA, MICA-K, MICA, ICA, ACO (Shelokar et al 2004; Niknam et al 2008a, 2008b; Niknam et al 2009; Bahmani Firouzi et al 2010), PSO (Kao et al 2008), SA (Sung \& Jin 2000), GA (Krishna \& Murty 1999), TS (Ng \& Wong 2002), HBMO (Fathian et al 2008) and $k$-means (Niknam et al 2008b; Niknam et al 2009; Niknam \& Amiri 2010; Sung \& Jin 2000; Bahmani Firouzi et al 2010) has been given in tables 3, 4,5 and 6 on four real-life data sets and table 7 on a manufactured data set for 100 random tails.

For Iris data (table 3 ) it is found that, the proposed clustering algorithm converges to the optimal value of 96.6554 in all times. The standard deviation of the fitness function for hybrid K-MICA clustering algorithm is zero. This means that proposed algorithm is very precise and reliable. In other words, it provides the optimum value and small standard deviation in comparison to those of other methods. 
Table 1. Values of parameters of each of five algorithms.

\begin{tabular}{|c|c|c|c|c|c|c|c|}
\hline \multicolumn{2}{|c|}{ Hybrid K-MICA } & \multicolumn{2}{|c|}{ K-MICA and MICA-K } & \multicolumn{2}{|c|}{ MICA } & \multicolumn{2}{|l|}{ ICA } \\
\hline Parameter & Value & Parameter & Value & Parameter & Value & Parameter & Value \\
\hline$N_{p o p}$ & 100 & $\mathrm{c} N_{p o p}$ & 100 & $N_{\text {pop }}$ & 100 & $N_{\text {pop }}$ & 100 \\
\hline$N_{\text {imp }}$ & 6 & $N_{i m p}$ & 6 & $N_{i m p}$ & 8 & $N_{\text {imp }}$ & 8 \\
\hline$\beta$ & 5 & $\beta$ & 5 & $\beta$ & 5 & $\beta$ & 5 \\
\hline$\xi$ & 0.05 & $\xi$ & 0.05 & $\xi$ & 0.05 & $\xi$ & 0.05 \\
\hline$\gamma$ & 0.7 & $\gamma$ & 0.7 & $\gamma$ & 0.7 & $\gamma$ & 0.7 \\
\hline \# iterations & 500 & \# iterations & 500 & $\#$ iterations & 500 & \# iterations & 500 \\
\hline \multicolumn{2}{|c|}{ HBMO } & \multicolumn{2}{|l|}{ SA } & \multicolumn{2}{|c|}{$\mathrm{ACO}$} & & \\
\hline Parameter & Value & Parameter & Value & Parameter & Value & & \\
\hline queens & 1 & $\begin{array}{l}\text { Probability } \\
\text { threshold }\end{array}$ & 0.98 & $\#$ ants & 50 & & \\
\hline \# drones & 150 & $\begin{array}{l}\text { Initial } \\
\text { temperature }\end{array}$ & 5 & $\begin{array}{l}\text { Probability } \\
\text { threshold for } \\
\text { maximum } \\
\text { trail }\end{array}$ & 0.98 & & \\
\hline $\begin{array}{l}\text { Capacity of } \\
\text { spermatheca }\end{array}$ & 50 & $\begin{array}{l}\text { Temperature } \\
\text { multiplier }\end{array}$ & 0.98 & $\begin{array}{l}\text { Local search } \\
\text { probability }\end{array}$ & 0.01 & & \\
\hline $\begin{array}{l}\text { Maximum } \\
\text { speed }\end{array}$ & $\begin{array}{l}\text { Randomly } \\
\in\left[\begin{array}{ll}0.51 & 1\end{array}\right.\end{array}$ & $\begin{array}{l}\text { Final } \\
\text { temperature }\end{array}$ & 0.01 & $\begin{array}{l}\text { Evaporation } \\
\text { rate }\end{array}$ & 0.01 & & \\
\hline $\begin{array}{l}\text { Minimum } \\
\text { speed }\end{array}$ & $\begin{array}{l}\text { Randomly } \\
\quad \in\left[\begin{array}{ll}0 & 1\end{array}\right]\end{array}$ & $\begin{array}{l}\text { Number of } \\
\text { Iterations } \\
\text { detect } \\
\text { steady stat }\end{array}$ & 100 & \# iterations & 1000 & & \\
\hline $\begin{array}{l}\text { Speed } \\
\text { reduction }\end{array}$ & 0.98 & \# iterations & 30000 & & & & \\
\hline Crossover & 1.5 & & & & & & \\
\hline \# iterations & 1000 & & & & & & \\
\hline \multicolumn{2}{|c|}{ GA } & \multicolumn{2}{|l|}{$\mathrm{TS}$} & \multicolumn{2}{|c|}{ PSO } & & \\
\hline Parameter & Value & Parameter & Value & Parameter & Value & & \\
\hline Population & 50 & Tabu list size & 25 & \# Swarm & $10 \times \mathrm{K} \times \mathrm{d}$ & & \\
\hline Crossover & 0.8 & $\begin{array}{l}\text { Number } \\
\text { of trial } \\
\text { solutions }\end{array}$ & 40 & $\mathrm{C}_{1}=\mathrm{C}_{2}$ & 2 & & \\
\hline Mutation Rate & 0.001 & $\begin{array}{l}\text { Probability } \\
\text { threshold }\end{array}$ & 0.98 & $\omega_{\min }$ & 0.5 & & \\
\hline \# iterations & 1000 & \# iterations & 1000 & $\begin{array}{l}\omega_{\max } \\
\# \text { iterations }(7)\end{array}$ & $\begin{array}{c}1 \\
500\end{array}$ & & \\
\hline
\end{tabular}

The best value obtained by hybrid K-MICA clustering algorithm for Wine data (table 4) is 16292.65 while the other algorithms fail to attain this value in any of their runs. The results presented in table 5 show that the worst result obtained by the proposed algorithm is better than the best result found by other methods. Not only the best result but also the worst result of the proposed algorithm is less than the best results of other methods, which indicates the effectiveness of the proposed algorithm to solve the clustering problem.

The results obtained on the CMC data set show that hybrid K-MICA converges to the global optimum of 5,693.9198 while the best solutions of K-MICA, MICA-K, MICA, ICA, PSO, 
Table 2. Simulation results of hybrid K-MICA algorithm parameters for Iris data set.

\begin{tabular}{lcccccccc}
\hline Case & $N_{\text {pop }}$ & $N_{\text {imp }}$ & $\beta$ & $\xi$ & $\gamma$ & Best solution & Worst solution & Average solution \\
\hline 1 & 150 & 12 & 20 & 0.5 & 0.8 & 96.6554 & 96.6554 & 96.6554 \\
2 & 150 & 8 & 20 & 0.1 & 0.7 & 96.6554 & 96.6554 & 96.6554 \\
3 & 120 & 4 & 15 & 0.05 & 0.6 & 96.6554 & 96.6554 & 96.6554 \\
4 & 120 & 12 & 15 & 0.5 & 0.7 & 96.6554 & 96.6554 & 96.6554 \\
5 & 100 & 8 & 10 & 0.1 & 0.6 & 96.6554 & 96.6554 & 96.6554 \\
6 & 100 & 4 & 10 & 0.05 & 0.5 & 96.6554 & 96.6554 & 96.6554 \\
7 & 80 & 8 & 5 & 0.5 & 0.5 & 96.6554 & 96.6554 & 96.6554 \\
8 & 80 & 4 & 5 & 0.1 & 0.4 & 96.6554 & 96.6554 & 96.6554 \\
9 & 30 & 8 & 1 & 0.05 & 0.4 & 96.9672 & 97.1304 & 96.9948 \\
10 & 30 & 4 & 1 & 0.5 & 0.3 & 96.8634 & 96.9716 & 96.9141 \\
\hline
\end{tabular}

Table 3. Results obtained by the algorithms for 100 different runs on Iris data.

\begin{tabular}{lcccc}
\hline Method & \multicolumn{3}{c}{ Function value } & Standard deviation \\
\cline { 2 - 4 } & $\mathrm{F}_{\text {best }}$ & $\mathrm{F}_{\text {average }}$ & $\mathrm{F}_{\text {worst }}$ & \\
\hline Hybrid K-MICA & 96.6554 & 96.6554 & 96.6554 & 0 \\
K-MICA & 96.6564 & 96.68344 & 96.7588 & 0.0359928 \\
MICA-K & 96.6556 & 96.66691 & 96.7071 & 0.018515 \\
MICA & 96.6562 & 96.6664 & 96.6919 & 0.0114455 \\
ICA & 96.6997 & 96.8466 & 97.0059 & 0.1114908 \\
PSO & 96.8942 & 97.2328 & 97.8973 & 0.347168 \\
SA & 97.4573 & 99.957 & 102.01 & 2.018 \\
TS & 97.365977 & 97.868008 & 98.569485 & 0.53 \\
GA & 113.986503 & 125.197025 & 139.778272 & 14.563 \\
ACO & 97.100777 & 97.171546 & 97.808466 & 0.367 \\
HBMO & 96.752047 & 96.95316 & 97.757625 & 0.531 \\
$K$-means & 97.333 & 106.05 & 120.45 & 14.6311 \\
\hline
\end{tabular}

Table 4. Results obtained by the algorithms for 100 different runs on Wine data.

\begin{tabular}{llccc}
\hline Method & \multicolumn{3}{c}{ Function value } & Standard deviation \\
\cline { 2 - 4 } & \multicolumn{1}{c}{$\mathrm{F}_{\text {best }}$} & $\mathrm{F}_{\text {average }}$ & $\mathrm{F}_{\text {worst }}$ & \\
\hline Hybrid K-MICA & 16292.65 & 16293.12 & 16293.81 & 0.4331 \\
K-MICA & 16293.85 & 16294.83 & 16296.5 & 0.924925 \\
MICA-K & 16293.6 & 16295 & 16296.8 & 0.992492 \\
MICA & 16293.9 & 16295.6 & 16296.94 & 1.002372 \\
ICA & 16295.24 & 16298.57 & 16304.61 & 2.934509 \\
PSO & $16,345.9670$ & $16,417.4725$ & $16,562.3180$ & 85.4974 \\
SA & $16,473.4825$ & $17,521.094$ & $18,083.251$ & 753.084 \\
TS & $16,666.22699$ & $16,785.45928$ & $16,837.53567$ & 52.073 \\
GA & $16,530.53381$ & $16,530.53381$ & $16,530.53381$ & 0 \\
ACO & $16,530.53381$ & $16,530.53381$ & $16,530.53381$ & 0 \\
HBMO & $16,357.28438$ & $16,357.28438$ & $16,357.28438$ & 0 \\
$K$-means & $16,555.68$ & 18,061 & $18,563.12$ & 793.213 \\
\hline
\end{tabular}


Table 5. Results obtained by the algorithms for 100 different runs on CMC data.

\begin{tabular}{lcccc}
\hline Method & \multicolumn{3}{c}{ Function value } & Standard deviation \\
\cline { 2 - 4 } & $\mathrm{F}_{\text {best }}$ & $\mathrm{F}_{\text {average }}$ & $\mathrm{F}_{\text {worst }}$ & \\
Hybrid K-MICA & $5,693.9198$ & $5,694.0012$ & 5694.224 & 0.018423 \\
K-MICA & $5,695.8547$ & $5,696.8659$ & 5698.0194 & 1.111793 \\
MICA-K & $5,694.8723$ & $5,695.5322$ & $5,697.50$ & 1.268275 \\
MICA & $5,699.2183$ & $5,705.1485$ & 5721.1779 & 7.397884 \\
ICA & $5,725.7062$ & $5,736.3663$ & 5752.9425 & 8.000562 \\
PSO & $5,700.9853$ & $5,820.9647$ & $5,923.2490$ & 46.959690 \\
SA & $5,849.0380$ & $5,893.4823$ & $5,966.9470$ & 50.867200 \\
TS & $5,885.0621$ & $5,993.5942$ & $5,999.8053$ & 40.84568 \\
GA & $5,705.6301$ & $5,756.5984$ & $5,812.6480$ & 50.3694 \\
ACO & $5,701.9230$ & $5,819.1347$ & $5,912.4300$ & 45.634700 \\
HBMO & $5,699.2670$ & $5,713.9800$ & $5,725.3500$ & 12.690000 \\
$K$-means & $5,842.20$ & $5,893.60$ & $5,934.43$ & 47.16 \\
\hline
\end{tabular}

Table 6. Results obtained by the algorithms for 100 different runs on Vowel data.

\begin{tabular}{lllcc}
\hline Method & \multicolumn{3}{c}{ Function value } & Standard deviation \\
\cline { 2 - 4 } & \multicolumn{1}{c}{$\mathrm{F}_{\text {best }}$} & \multicolumn{1}{c}{$\mathrm{F}_{\text {average }}$} & $\mathrm{F}_{\text {worst }}$ & \\
\hline Hybrid K-MICA & $148,967.2408$ & $148,978.8291$ & $148,999.3829$ & 10.92318 \\
K-MICA & $149,279.9922$ & $149,596.3367$ & $149,955.0055$ & 280.0121 \\
MICA-K & $149,244.6165$ & $149,737.0533$ & $150,837.4077$ & 638.8130 \\
MICA & $149,332.1800$ & $150,204.1368$ & $150,982.4586$ & 733.0634 \\
ICA & $150,991.6147$ & $151,547.0511$ & $152,735.1651$ & 704.0907 \\
PSO & $148,976.0152$ & $151,999.8251$ & $158,121.1834$ & $28,813.4692$ \\
SA & $149,370.4700$ & $161,566.2810$ & $165,986.4200$ & $2,847.08594$ \\
TS & $149,468.268$ & $162,108.5381$ & $165,996.4280$ & $2,846.23516$ \\
GA & $149,513.735$ & $159,153.498$ & $165,991.6520$ & $3,105.5445$ \\
ACO & $149,395.602$ & $159,458.1438$ & $165,939.8260$ & $3,485.3816$ \\
HBMO & $149,201.632$ & $161,431.0431$ & $165,804.671$ & $2,746.0416$ \\
$K$-means & $149,422.26$ & $159,242.89$ & $161,236.81$ & 916 \\
\hline
\end{tabular}

Table 7. Results obtained by the algorithms for 100 different runs on manufactured data set.

\begin{tabular}{llccc}
\hline Method & \multicolumn{3}{c}{ Function value } & \multirow{2}{*}{ Standard deviation } \\
\cline { 2 - 4 } & \multicolumn{1}{c}{$\mathrm{F}_{\text {best }}$} & \multicolumn{1}{c}{$\mathrm{F}_{\text {average }}$} & $\mathrm{F}_{\text {worst }}$ & \\
\hline Hybrid K-MICA & 15187.5385 & 15187.6238 & 15187.954 & 0.104757 \\
K-MICA & 15191.0035 & 15880.054 & 16569.105 & 0.666306 \\
MICA-K & 15197.518 & 15211.856 & 15226.194 & 0.26355 \\
MICA & 15188.7359 & 15775.317 & 16363.096 & 4.102628 \\
ICA & 15259.5165 & 15551.466 & 158434155 & 2.118549 \\
\hline
\end{tabular}




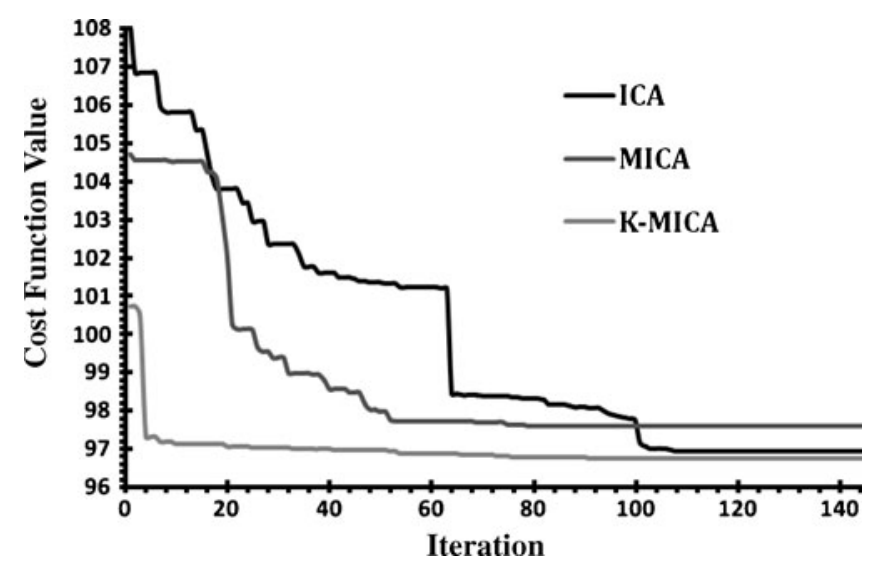

Figure 8. Convergence characteristic of ICA, MICA and K-MICA for the best solutions on Iris data.

SA, TS, GA, ACO, HBMO and $k$-means are 5695.8547, 5694.8723, 5699.2183, 5725.7062, $5700.9853,5849.0380,5885.0621,5705.6301,5701.9230,5699.2670$ and 5842.20. The standard deviation of the fitness function for this algorithm is 0.018423 , which is significantly less than other methods. Table 6 shows the results obtained on Vowel data set. It can be seen from table 5 that the proposed technique provided better results than those obtained by other methods. Therefore, the proposed method is more robust and practically applicable to real systems.

Table 7 shows the results of algorithms on the manufactured data set. The optimum value obtained by the proposed algorithm is 15187.5385 . Noticeably other algorithms fail to attain this value even once within 100 runs.

Figures 8-15 show the convergence characteristics of hybrid K-MICA, K-MICA, ICA-K, MICA, ICA, and $k$-means for the best solutions on Iris, Wine and Manufactured data set.

Simulation results of the figures show that $k$-means algorithm converges faster than the other algorithms but converges prematurely to a local optimum. For the Iris data set, hybrid K-MICA converges to the global optimum after 100 iterations while K-MICA, MICA-K, MICA and ICA converge to the global optimum in about $95,85,80$ and 110 iterations. The convergence characteristics of these algorithms for Wine data show that combining $k$-means with MICA converges

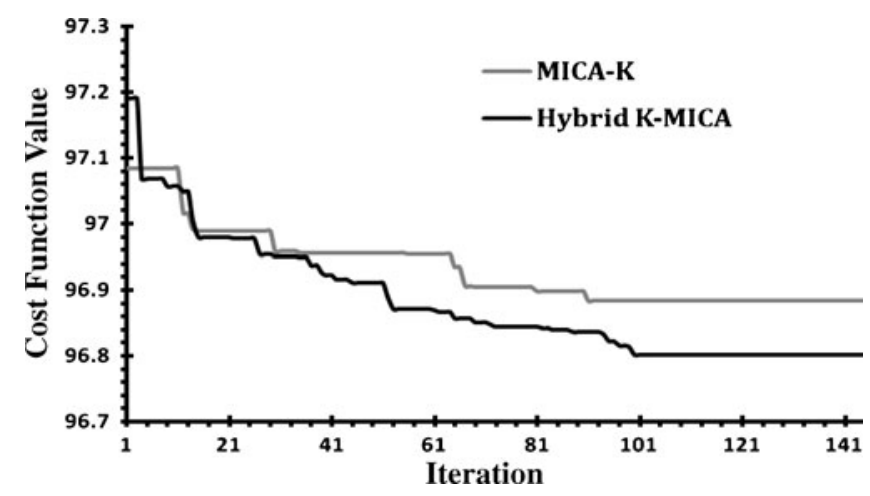

Figure 9. Convergence characteristic of MICA-K and hybrid K-MICA for the best solutions on Iris data. 


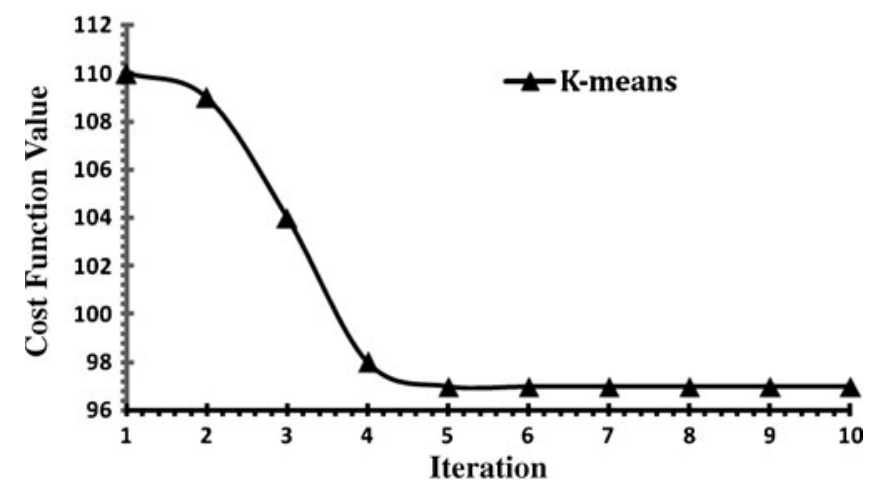

Figure 10. Convergence characteristic of $k$-means for the best solutions on Iris data.

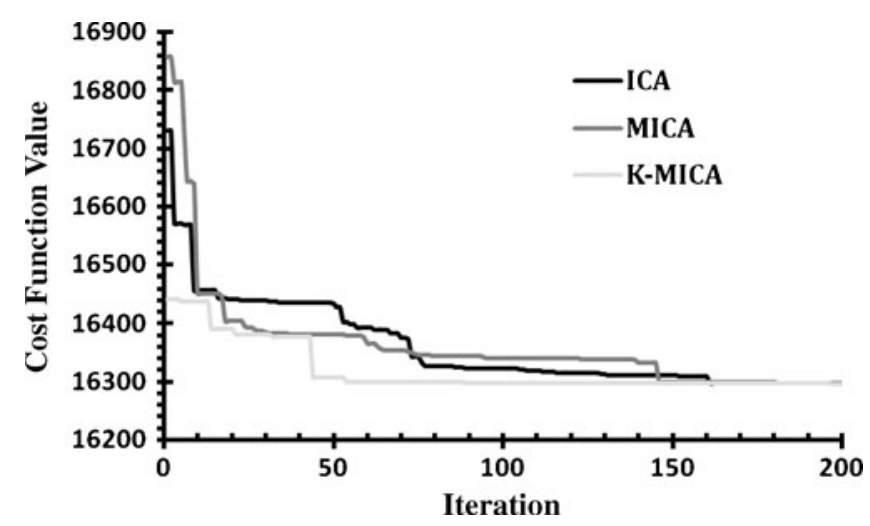

Figure 11. Convergence Characteristic of ICA, MICA and K-MICA for the best solutions on Wine data.

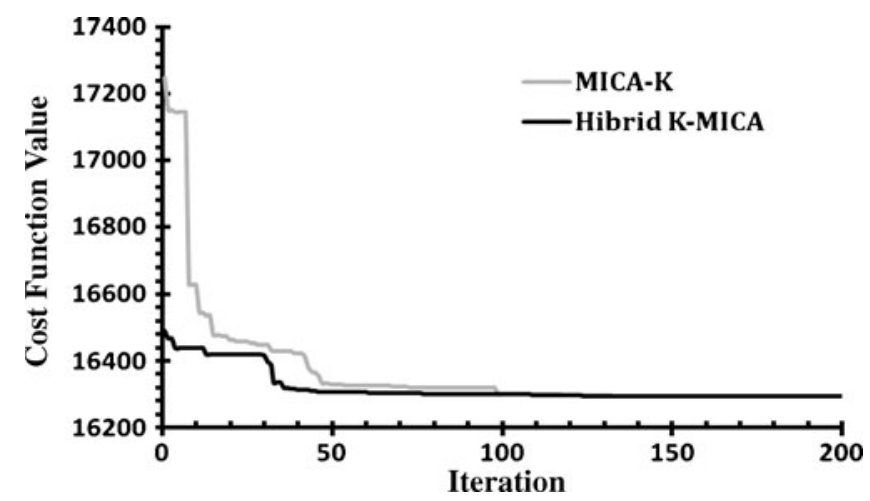

Figure 12. Convergence characteristic of MICA-K and hybrid K-MICA for the best solutions on Wine data. 


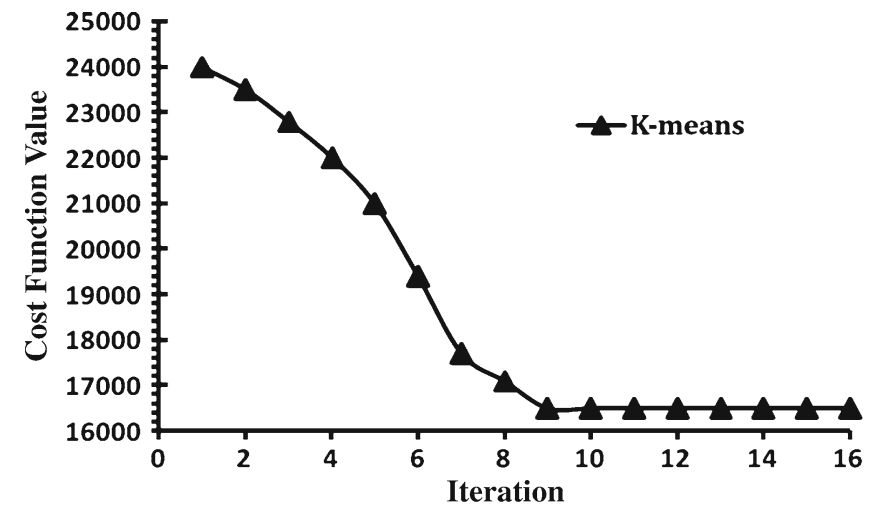

Figure 13. Convergence characteristic of $K$-means for the best solutions on Wine data.

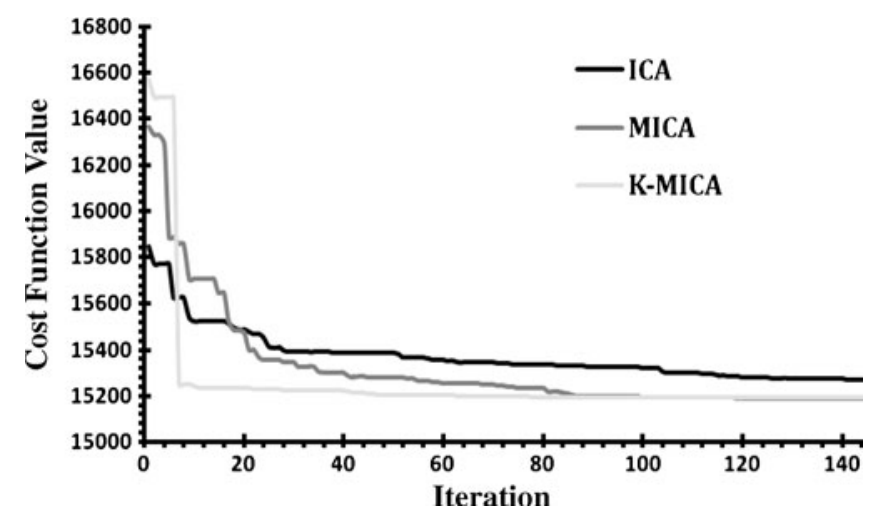

Figure 14. Convergence characteristic of ICA, MICA and K-MICA for the best solutions on a manufactured data.

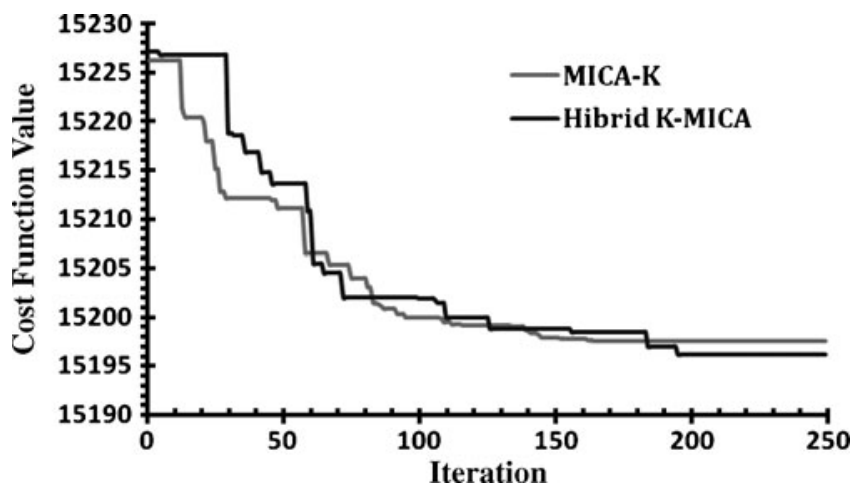

Figure 15. Convergence characteristic of MICA-K and hybrid K-MICA for the best solutions on a manufactured data. 
to better results. While ICA and MICA converge to the global solution after 161 and 150 iterations, MICA-K, K-MICA and hybrid K-MICA converge after 140, 70 and 60 iterations. The convergence characteristics of these algorithms for Manufactured data set show that combining $k$-means with MICA converges to better results. While ICA and MICA converge to the global solution after 150 and 90 iterations, MICA-K, K-MICA and hybrid K-MICA converge after 170,50 and 200 iterations. In all the data sets, while hybrid K-MICA needs more iterations than MICA-K and K-MICA algorithms, the best cost function will be achieved by it.

\section{Conclusion}

In this paper, a new hybrid evolutionary algorithm is proposed to find optimal or near optimal solutions for clustering problems of allocating $N$ objects to $k$ clusters. The proposed algorithm is based on a combination of ICA, SA and $k$-means algorithms called hybrid K-MICA. In this new algorithm, first, we use EM algorithm to find numbers of clusters and then apply $k$-means for each empire to select the best empires just before competition started by MICA. The algorithm is tested on several well-known real-life data sets. The experimental results indicate that the proposed optimization algorithm is at least comparable to the other algorithms in terms of function evaluations and standard deviations. In addition, the proposed algorithm is applicable when the number of clusters is not known a priori. The results illustrate that the proposed hybrid K-MICA optimization algorithm can be considered as a viable and an efficient heuristic.

Authors thank Dr. Behrooz Safarinejadian and Mrs. Shahinpour for their technical discussions.

\section{References}

Anderson E 1935 The irises of the Gaspe Peninsula. Bulletin of the American Iris Society 59: 2-5

Atashpaz-Gargari E, Lucas C 2007a Imperialist competitive algorithm: an algorithm for optimization inspired by imperialistic competition. IEEE Congress on Evolutionary Computation 4661-4667

Atashpaz-Gargari E, Lucas C 2007b Designing an optimal PID controller using Colonial Competitive Algorithm, First Iranian Joint Congress on Fuzzy and Intelligent Systems. Mashhad. Iran

Atashpaz-Gargari E, Hashemzadeh F, Lucas C 2008a Designing MIMO PID controller using colonial competitive algorithm. Proceeding of IEEE CEC 2008, within IEEE WCCI 2008 1929-1934

Atashpaz-Gargari E, Hashemzadeh F, Rajabioun R, Lucas C 2008b Colonial competitive algorithm: a novel approach for PID controller design in MIMO distillation column process. Int. J. Intelligent Computing and Cybernetics (IJICC) 1(3): 337-355

Bahmani Firouzi B, Sha Sadeghi M, Niknam T 2010 A new hybrid algorithm based on PSO, SA, and $k$-means for cluster analysis. Int. J. Innovative Computing Information and Control. 6(4): 1-10

Cao D N, Krzysztof J Cios 2008 GAKREM: a novel hybrid clustering algorithm. Information Sciences 178: 4205-4227

Fathian M, B Amiri, Maroosi Ali 2008 A honey-bee mating approach on clustering. The International Journal of Advanced Manufacturing Technology 43(9-10): 809-821

Figueiredo M A T, Jain A K 2002 Unsupervised learning of finite mixture models. IEEE Transactions on Pattern Analysis and Machine Intelligence 24(3): 381-396

Fisher R A 1936 The use of multiple measurements in taxonomic problems. Annals of Eugenics 7: 179-188

Huan Min Xu, Dong Bo Li 2008 A clustering-based modeling scheme of the manufacturing resources for process planning. The International Journal of Advanced Manufacturing Technology 38(1-2): 154-162

Jasour A M, Atashpaz Gargari E, Lucas C 2008 Vehicle fuzzy controller design using imperialist competitive algorithm. Second Iranian Joint Congress on Fuzzy and Intelligent Systems. Tehran, Iran 
Kao Y T, Zahar E, I. Kao W 2008 A hybridized approach to data clustering. Expert Systems with Applications 34(3): 1754-1762

Krishna K, Murty M 1999 Genetic $k$-means algorithm. IEEE Transactions on Systems. Man and Cybernet, Part B: Cybernet 29: 433-439

Laszlo M, Mukherjee S 2007 A genetic algorithm that exchanges neighboring centers for $k$-means clustering. Pattern Recognition Letters 28(16): 2359-2366

Lloyd 1982 Least square quantization in PCM. IEEE Transactions on Information Theory 28(2): 129-137

MacQueen J B 1967 Some Methods for classification and Analysis of Multivariate Observations. Proceedings of 5th Berkeley Symposium on Mathematical Statistics and Probability 281-297

Morales A K, Erazo F R 2009 A search space reduction methodology for data mining in large data bases. Engineering Application of Artificial Intelligence 22(1): 92-100

Mualik U, Bandyopadhyay S 2000 Genetic algorithm-based clustering technique. Journal of Pattern Recognition Letters 33: 1455-1465

$\mathrm{Ng}$ M K, Wong J C 2002 Clustering categorical data sets using tabu search techniques. Journal of Pattern Recognition Letters 35(12): 2783-2790

Niknam T, Olamaie J, Amiri B 2008a A hybrid evolutionary algorithm based on ACO and SA for cluster analysis. Journal of Applied Science 8(15): 2695-2702

Niknam T, Bahmani Firouzi B, Nayeripour M 2008b An efficient hybrid evolutionary algorithm for cluster analysis. World Applied Sciences Journal 4(2): 300-307

Niknam T, Amiri B, Olamaie J, Arefi A 2009 An efficient hybrid evolutionary optimization algorithm based on PSO and SA for clustering. Journal of Zhejiang University of Science A 10(4):512-519

Niknam T, Amiri B 2010 An efficient hybrid approach based on PSO, ACO and $k$-means for cluster analysis. Journal of Applied Soft Computing 10(1): 183-197

Rajabioun R, Hashemzadeh F, Atashpaz-Gargari E 2008a Colonial competitive algorithm: a novel approach for PID controller design in MIMO distillation column process. Int. J. Intelligent Computing and Cybernetics 1(3): 337-355

Rajabioun R, Hashemzadeh F, Atashpaz-Gargari E, Mesgari B, Rajaiee Salmasi F 2008b Identification of a MIMO evaporator and Its decentralized PID controller tuning using colonial competitive algorithm. The International Federation of Automatic Control Congress. Seoul Korea:9952-9957

Roshanaei M, Atashpaz-Gargari E, Lucas C 2008 Adaptive beamforming using colonial competitive algorithm. 2nd International Joint Conference on Computational Engineering. Vancouver. Canada.

Safarinejadian B, Menhaj Mohammad B, Karrari Mehdi 2010 Distributed unsupervised gaussian mixture learning for density estimation in sensor networks. IEEE Transactions on Instrumentation and Measurement 59(9): 2250-2260

Shelokar P S, Jayaraman V K, Kulkarni B D 2004 An ant colony approach for clustering. Analytica Chimica Acta 509(2): 187-195

Sung C S, Jin H W 2000 A tabu-search-based heuristic for clustering. Pattern Recognition Letters 33(5): 849-858

Tibshirani R, Walther G, Hastie T 2001 Estimating the number of clusters in a data set via the gap statistic. J. Statistical Soc., Series B 63(2):411-423

Zalik K R 2008 An efficient $k$-means clustering algorithm. Pattern Recognition Letters 29: 1385-1391 